\title{
INFRARED TRANSITIONS OF DIATOMIC MOLECULES AND THE TELLURIC LINES
}

\author{
Daniel Lozano-Gómez ${ }^{1}$, Alejandro García-Varela ${ }^{2}$, and Beatriz Sabogal ${ }^{2}$ \\ Received September 19 2019; accepted May 132020
}

\begin{abstract}
We present a simple model, as a pedagogical exercise, in order to obtain infrared transition energies for heteronuclear diatomic molecules, based on rotational and vibrational Hamiltonians. Our calculations allow us to identify CO absorption lines that are present in the atmosphere spectra acquired in astronomical observatories.
\end{abstract}

\section{RESUMEN}

Presentamos un modelo sencillo, a manera de ejercicio pedagógico, con el fin de obtener las energías de transición en infrarojo de moléculas heteronucleares, basados en Hamiltonianos de rotación y vibración. Nuestros cálculos permiten identificar líneas de absorción de CO presentes en los espectros de la atmósfera, adquiridos en observatorios astronómicos.

Key Words: line: identification — methods: analytical — molecular data

\section{INTRODUCTION}

Infrared (IR) spectroscopy is a useful tool in studying systems at temperatures around $10^{2} \mathrm{~K}$. Objects at these temperatures such as protoplanetary, accretion, and protostellar disks, significantly radiate in this region of the electromagnetic spectrum. Commonly, IR studies are conducted in ground-based observatories; thus, the observed spectrum presents lines of both the astronomical source and the terrestrial atmosphere. Therefore, including the atmospheric absorption is a crucial step towards any study involving IR astronomical spectra in order to avoid misidentifications with the atmospheric absorption lines. This implies dealing with the atmosphere, which is made up of a large quantity of gas molecules at temperatures of the same order as those of the observed sources.

The Earth's atmosphere is mostly composed of $\mathrm{N}_{2}(78.09 \%)$ and $\mathrm{O}_{2}(20.95 \%)$, both accounting for almost $99 \%$ of the average composition of the dry atmosphere (Cox 2000). These homonuclear ${ }^{3}$ diatomic molecules are inactive in the IR because of their null electric dipole moment. In fact, ac-

\footnotetext{
${ }^{1}$ Department of Physics and Astronomy, University of Waterloo, Canada.

${ }^{2}$ Universidad de los Andes, Departamento de Física, Colombia.

${ }^{3} \mathrm{~A}$ molecule composed of only one type of chemical element.
}

cording to Gross selection rules (Atkins \& de Paula 2014), fundamental vibrational transitions are forbidden for these molecules and no IR contribution is observed. On the other hand, atmospheric heteronuclear molecules such as $\mathrm{CO}, \mathrm{CO}_{2}$ and $\mathrm{H}_{2} \mathrm{O}$, are some of the ones responsible for the major features of the IR absorption atmospheric spectrum, due to rotational and vibrational transitions.

The spectrum theory of diatomic molecules is presented in detail in classical books (Kronig 1930; Herzberg 1950) and also in physical chemistry texts (Atkins \& de Paula 2014). As the atmosphere is composed of diatomic and polyatomic gases, aerosols, dust, pollutants and other constituents, studying its spectrum is a complex task. The variable concentration of $\mathrm{CO}$ and $\mathrm{CO}_{2}$ due to its major sources: anthropogenic, biological, oceanic, combustion, photochemical, and the strongly variable concentration of the water vapor due to atmospheric and oceanic conditions, cause the IR spectrum of the terrestrial atmosphere, called the telluric ${ }^{4}$ spectrum, to change in time scales of seconds and up to months. Currently, simulating the atmospheric molecular transitions requires the development of complex algorithms ${ }^{5}$ which consider

\footnotetext{
${ }^{4}$ In latin tellus mean "Earth".

${ }^{5}$ In this regard, HITRAN is one of the databases with greater use. https://hitran.org/.
} 
the different quantum interactions present in these molecules (Rudolf et al. 2016; Ulmer-Moll et al. 2019, and references therein).

The aim of the present work is to obtain, as a pedagogical exercise, analytical expressions for the IR transition energies of heteronuclear atmospheric molecules using undergraduate quantum mechanics, and to show that these transitions produce bands in the IR spectra. The organization of this study is as follows: in $\S 2$ we present the Hamiltonians that describe the simplest approximation to the vibrational and rotational transitions of molecules. In $\S 3$ we compute vibration and rotation transitions, using a more realistic Hamiltonian through a Morse potential. In $\S 4$ we present our results, showing that CO IR transitions form bands. Finally, in the last section we give the main conclusions of this work.

\section{MODELING VIBRATION AND ROTATION OF DIATOMIC MOLECULES}

To describe the energy spectrum of a diatomic molecule the vibrational and rotational degrees of freedom are considered. Studies of degrees of freedom considering complex models are discussed by Stuart (2004). In this section, we present the vibrational and rotational Hamiltonians for a system without and with an interaction term between each degree of freedom.

\subsection{Vibrational Hamiltonian}

A diatomic gas molecule has a spectrum originated in part by the vibrational motion. Because these vibrations are not completely harmonic, there must be introduced correction terms that account for the non-harmonic behavior of the molecule. As a first approximation, one can add to the potential an extra term proportional to $\hat{x}^{3}$. This procedure is similar to taking a smooth potential and performing a Taylor expansion around its minimum value, as made frequently in the quantum mechanics text books (see for example Cohen-Tannoudji et al. 1977). This correction is known as the anharmonic oscillator potential. Following this approximation, the vibrational Hamiltonian can be written as:

$$
\hat{H}_{v i b}=\frac{\hat{p}^{2}}{2 \mu}+\frac{1}{2} \mu \omega^{2} \hat{x}^{2}+\frac{1}{2} G_{1} \mu \omega^{2} \hat{x}^{3}+\cdots,
$$

where $\mu$ is the reduced mass of the system, $\omega$ is the oscillation frequency and $G_{1}$ is a small parameter related to the first correction of the potential. Higher order correction terms are described by the factors $G_{i>1}$.

Remembering the results for the regular quantum harmonic oscillator, we use the creation $\hat{a}$, annihilation $\hat{a}^{\dagger}$ and number $\hat{N}=\hat{a}^{\dagger} \hat{a}$ operators, with their usual commutation relations. The canonical definition of this operators is given by the following relations:

$$
\begin{gathered}
\hat{a}=\sqrt{\frac{\mu \omega}{2 \hbar}}\left(\hat{x}+\frac{i}{\mu \omega} \hat{p}\right), \quad \hat{a}^{\dagger}=\sqrt{\frac{\mu \omega}{2 \hbar}}\left(\hat{x}-\frac{i}{\mu \omega} \hat{p}\right), \\
\hat{x}=\sqrt{\frac{\hbar}{2 \mu \omega}}\left(\hat{a}+\hat{a}^{\dagger}\right) .
\end{gathered}
$$

For the anharmonic Hamiltonian, the term corresponding to $\hat{x}^{3}$ can be computed:

$$
\begin{aligned}
\left(\hat{a}+\hat{a}^{\dagger}\right)^{3}= & \left(\hat{a}+\hat{a}^{\dagger}\right)\left(\hat{a}^{2}+\left(\hat{a}^{\dagger}\right)^{2}+1+2 \hat{N}\right) \\
= & \hat{a}^{3}+\left(\hat{a}^{\dagger}\right)^{3}+\hat{a}+2 \hat{a}^{\dagger}+\left(\hat{a}+\hat{a}^{\dagger}\right)(2 \hat{N}) \\
& +\hat{N}\left(\hat{a}+\hat{a}^{\dagger}\right) .
\end{aligned}
$$

Hence, the cubic term given by equation (1) can be rewritten in the following way:

$$
\begin{aligned}
& \frac{1}{2} G_{1} \mu \omega^{2} \hat{x}^{3}=\left[\frac{1}{2} G_{1} \mu \omega^{2}\left(\frac{\hbar}{2 \mu \omega}\right)^{3 / 2}\right]\left(\hat{a}^{3}+\left(\hat{a}^{\dagger}\right)^{3}+\hat{a}\right. \\
& \left.+2 \hat{a}^{\dagger}+\left(\hat{a}+\hat{a}^{\dagger}\right)(2 \hat{N})+\hat{N}\left(\hat{a}+\hat{a}^{\dagger}\right)\right) .
\end{aligned}
$$

To continue, the action of the correction term over $|n\rangle$ is obtained:

$$
\begin{aligned}
\hat{x}^{3}|n\rangle \propto & \sqrt{n(n-1)(n-2)}|n-3\rangle\rangle \\
& +\sqrt{(n+1)(n+2)(n+3)}|n+3\rangle \\
& +(\sqrt{n}+2 n \sqrt{n}+(n-1) \sqrt{n})|n-1\rangle \\
+(2 \sqrt{n+1} & +2 n \sqrt{n+1}+(n+1) \sqrt{n+1})|n+1\rangle .
\end{aligned}
$$

Given the small size of the correction $G_{1}$ term, perturbation theory can be applied. Notice that this Hamiltonian does not present a first order correction to its energy levels. We invite the readers to calculate the second order perturbation theory correction (see Cohen-Tannoudji et al. 1977) to find the following results for the energy:

$$
\begin{aligned}
E & \propto \frac{n(n-1)(n-2)}{3 \hbar \omega}-\frac{(n+1)(n+2)(n+3)}{3 \hbar \omega} \\
& +\frac{9 n^{3}}{\hbar \omega(n-n+1)}+\frac{9(n+1)^{3}}{\hbar \omega(n-n-1)} \\
E & \propto-\left[\frac{1}{\hbar \omega}\right]\left(30 n^{2}+30 n+11\right) .
\end{aligned}
$$


Plugging back these correction terms into the corrected energy term, a new energy spectrum is found.

$$
\begin{aligned}
\hat{H}_{v i b}|n\rangle= & \hbar \omega\left(n+\frac{1}{2}\right)|n\rangle \\
- & {\left[\frac{1}{\hbar \omega}\right]\left(\frac{1}{2} G_{1} \mu \omega^{2}\left(\frac{\hbar}{2 \mu \omega}\right)^{3 / 2}\right)^{2} } \\
& \left(30 n^{2}+30 n+11\right)|n\rangle .
\end{aligned}
$$

This Hamiltonian describes the degrees of freedom corresponding to the vibrational nature of molecules.

\subsection{Rotational Hamiltonian}

When a molecule rotates, the centrifugal force pulls the atoms apart causing an increase in its moment of inertia and decreasing the rotational constant associated to this energy term. This effect couples both types of interactions, vibration and rotation. In order to understand this interaction, the following analogy can be useful: a pair of masses (atoms of a diatomic molecule) are attached at the ends of a spring, so that the masses can vibrate along the line between them. The spring is fixed at some point to the center of a rotating disk. The system has no friction. Due to rotation, the Coriolis force deflects the masses, changing the vibration frequency of the spring, reflecting an interaction between rotation and vibration in this mechanical system. This is analogous to the interaction between rotation and vibration in diatomic molecules.

Returning to the effect of the centrifugal force on the coupling of the rotation and vibration interactions and, therefore, on the rotational Hamiltonian, a term named centrifugal diatomic distortion is defined as

$$
D=\frac{4}{\omega^{2}}\left(\frac{B}{h c}\right)^{3}, \quad \text { with } B=\frac{\hbar^{2}}{2 \mu r^{2}} .
$$

The $r$ variable describes the inter-nuclear distance between the atoms composing the molecule. The rotational Hamiltonian is now the following:

$$
\hat{H}_{\text {rot }}=\frac{1}{2 I} \hat{L}^{2}+D \hat{L}^{4},
$$

where $\hat{L}$ is the angular momentum operator $(\hat{L}=\hat{r} \times \hat{p})$ and $I$ is the moment of inertia. The corrected Hamiltonian in equation (4) shares the same eigenstates $|l, m\rangle$ that are used in the rotational uncorrected Hamiltonian, where $D=0$. Thus the action of the Hamiltonian over these eigenstates produces:

$$
\hat{H}_{\text {rot }}|l, m\rangle=B l(l+1)|l, m\rangle+l^{2}(l+1)^{2} D|l, m\rangle .
$$

\section{HAMILTONIANS FOR DIATOMIC MOLECULES}

\subsection{Model 1: Combined Hamiltonian}

Before using the vibration and rotation Hamiltonians given by equations (1) and (4), it is necessary to find an appropriate basis which diagonalizes the Hamiltonians in the corresponding Hilbert space. If these Hamiltonians are defined as $\hat{H}_{\text {rot }}\left(L_{i}\right)$ and $\hat{H}_{v i b}\left(p_{i}, x_{i}\right)$, we can ensure the commutation relation $\left[\hat{H}_{\text {rot }}, \hat{H}_{v i b}\right]=0$. Hence we can simply add both terms and describe the total eigenvectors as a tensor product $|n, l\rangle=|n\rangle \otimes|l\rangle$. The quantum number $m$ has been left out since the total Hamiltonian does not present a dependency on it. The energy of such a system is given by:

$$
\begin{aligned}
& \hat{H}_{\text {total }}|n, l\rangle=\left(\hat{H}_{\text {rot }}+\hat{H}_{v i b}\right)|n, l\rangle \\
& =\left[\hbar \omega\left(n+\frac{1}{2}\right)-\frac{G_{1}^{2} \hbar^{2}}{32 \mu}\left(30 n^{2}+30 n+11\right)\right. \\
& \left.+B l(l+1)+l^{2}(l+1)^{2} D\right]|n, l\rangle .
\end{aligned}
$$

This description supposes that the movement of the rotation and the vibration are described in the same coordinates where the Hamiltonians commute. If the vibrations were considered along a different direction, these would affect the separation radius of the molecule, changing the moment of inertia of the system. These corrections cannot be ignored and correction terms must be added. This coupling is studied in the following section.

By taking into account both interaction terms, rotation and vibration, a net Hamiltonian can be studied. This Hamiltonian is fully described by the quantum numbers $n$ and $l$. This fact is a consequence of the commutation relation $\left[\hat{H}_{r o t}, \hat{H}_{v i b}\right]=0$, which states that a common basis describes the eigenstates of both terms. Through this identification, the energy levels are found to be:

$$
\begin{aligned}
E_{n, l} & =\hbar \omega\left(n+\frac{1}{2}\right)-\frac{G_{1}^{2} \hbar^{2}}{32 \mu}\left(30 n^{2}+30 n+11\right) \\
& +B l(l+1)+l^{2}(l+1)^{2} D
\end{aligned}
$$

Tables 1 and 2 give the vibrational and rotational transitions for an initial state $|n, l\rangle$. The energy transitions displayed above only show a change of state in a single Hamiltonian (vibrational or rotational). All the other transitions are described by the 
TABLE 1

\section{ALLOWED TRANSITIONS FOR VIBRATIONAL HAMILTONIAN}

\begin{tabular}{cl}
\hline$\Delta n(\Delta l=0)$ & \multicolumn{1}{c}{$\Delta E=E_{f}-E_{i}$} \\
\hline$\Delta n=-2$ & $-2 \hbar \omega+\left(G_{1}^{2} \hbar^{2} / 32 \mu\right)(-120 n+60)$ \\
$\Delta n=-1$ & $-\hbar \omega+\left(G_{1}^{2} \hbar^{2} / 32 \mu\right)(60 n)$ \\
$\Delta n=+1$ & $+\hbar \omega-\left(G_{1}^{2} \hbar^{2} / 32 \mu\right)(60 n+60)$ \\
$\Delta n=+2$ & $+2 \hbar \omega+\left(G_{1}^{2} \hbar^{2} / 32 \mu\right)(120 n+180)$ \\
\hline
\end{tabular}

combination of transitions between both quantum numbers given by the selection rules $\Delta n= \pm 1, \pm 2$ and $\Delta l= \pm 1, \pm 2$.

Notice that each initial state $|n, l\rangle$ has 24 possible final states given the corrections proposed by this model. It is worth mentioning that this correction is very restrictive as the rotation is fully confined to one direction. Moreover, the energy constant associated with this rotational degree of freedom is greater than that found in any other direction.

\subsection{Model 2: Morse Potential}

The Morse potential is commonly used to describe the vibrational behavior of diatomic molecules. Its asymptotic behavior, given by the maximum energy of vibrations (the dissociation energy, $D_{E}$, related to the depth of the potential well) more accurately describes the physics of diatomic molecules than the quantum harmonic oscillator.

$$
V(\mathbf{r})=D_{E}\left(1-e^{-a\left(r-r_{0}\right)}\right)^{2}
$$

In the above expression, $r_{0}$ is the equilibrium bond distance and $a$ is a constant related to the strength of the bond. By using this expression, the Schrödinger equation can be perturbatively solved obtaining the following $n$-dependent energy spectrum

$$
E_{n}=\hbar \omega\left(n+\frac{1}{2}\right)-\frac{\hbar^{2} \omega^{2}}{4 D_{E}}\left(n+\frac{1}{2}\right)^{2}
$$

\subsection{Rotation-Vibration Interaction}

In $\S 3.1$ we have described the energy spectrum of a diatomic molecule when both vibrations and rotations are present in the system. In such a way, the Hamiltonian can be split into two non-interacting terms, which share a common basis. However, this is not the general case as vibrations can affect the way the rotational spectrum is defined. This effect can be understood by considering the moment of inertia of a single molecule: given the vibration of the molecule, the length of the bond separating the molecules will change. Therefore, in order to introduce the radial separation $(r)$, the constants $B$ and $D$ appearing in the rotational Hamiltonian are now considered as functions of this variable:

$$
\begin{gathered}
B(r)=\frac{\hbar}{4 \pi c \mu r^{2}}, \\
D(r)=\frac{\hbar^{3}}{4 \pi c k \mu^{2} r^{6}} .
\end{gathered}
$$

Under the assumption that the vibration frequency is much greater than the rotational frequency, the average separation radius can be computed, and the effective separation can be described. To compute this effective separation, we consider the average radius in the wave function describing the oscillatory movement:

$$
\langle r\rangle=\int \psi_{v i b}^{\dagger} r \psi_{v i b} d r
$$

Following the same procedure, the quantities $B(r)$ and $D(r)$ are defined through their mean values in a full period of oscillation:

$$
\begin{aligned}
\left\langle\frac{1}{r^{2}}\right\rangle & =\int \psi_{v i b}^{\dagger} \frac{1}{r^{2}} \psi_{v i b} d r, \\
\left\langle\frac{1}{r^{6}}\right\rangle & =\int \psi_{v i b}^{\dagger} \frac{1}{r^{6}} \psi_{v i b} d r .
\end{aligned}
$$

Through this approximation, one obtains the following new constants related to the unperturbed terms, $B_{e}$ and $D_{e}$, presented in $\S 2.2$ for the rotational Hamiltonian:

$$
\begin{aligned}
B_{n} & =\langle B(r)\rangle=B_{e}-\alpha_{e}\left(n+\frac{1}{2}\right), \\
D_{n}(r) & =\langle D(r)\rangle=D_{e}-\beta_{e}\left(n+\frac{1}{2}\right),
\end{aligned}
$$

where the subindex $e$ refers to the constants given in the equation (4). The new constants, $\alpha_{e}$ and $\beta_{e}$, are the ones which introduce the interaction of these two Hamiltonians. These values have been reported by Le Floch (1991).

\subsection{Rotational and Vibrational Hamiltonian with Morse Potential}

To better describe the energy spectrum of a diatomic molecule, we should use a total Hamiltonian that includes a term related to the Morse potential (given in 
TABLE 2

ALLOWED TRANSITIONS FOR ROTATIONAL HAMILTONIAN

\begin{tabular}{cc}
\hline$\Delta n, \Delta l$ & $\Delta E=E_{f}-E_{i}$ \\
\hline$\Delta n=0, \Delta l=-2$ & $B(-4 l+2)+D\left(-10 l^{3}+11 l^{2}-12 l+4\right)$ \\
$\Delta n=0, \Delta l=-1$ & $-2 B l-4 D l^{3}$ \\
$\Delta n=0, \Delta l=+1$ & $B(2 l+2)+D\left(4 l^{3}+12 l^{2}+12 l+4\right)$ \\
$\Delta n=0, \Delta l=+2$ & $B(4 l+6)+D\left(12 l^{3}+47 l^{2}+72 l+36\right)$ \\
\hline
\end{tabular}

equation 8) and other term related to the rotational and vibrational coupling (involving the expressions given in equation 13). Obtaining this total Hamiltionian, $\hat{H}_{\text {total }}=\hat{H}_{\text {morse }}+\hat{H}_{\text {rot }}$ is left as an exercise for the reader, as well as the equation for energy in terms of the quantum numbers $\mathrm{n}$ and $\mathrm{l}$ :

$$
\begin{aligned}
E_{n, l}= & \hbar \omega\left(n+\frac{1}{2}\right)-\frac{\hbar^{2} \omega^{2}}{4 D_{E}}\left(n+\frac{1}{2}\right)^{2} \\
& +B_{n} l(l+1)+D_{n} l^{2}(l+1)^{2},
\end{aligned}
$$

where the first two terms are the given by equation (9) and the last two terms are the rotationalvibrational energy discussed in $\S 3.3$. Reordering terms, we obtain:

$$
\begin{aligned}
E_{n, l}= & {\left[\hbar \omega\left(n+\frac{1}{2}\right)+B_{e} l(l+1)\right] } \\
& -\left[\hbar^{2} \omega_{\chi}\left(n+\frac{1}{2}\right)^{2}+D_{e} l^{2}(l+1)^{2}\right] \\
& -\left(n+\frac{1}{2}\right)\left(\alpha_{e} l(l+1)+\beta_{e} l^{2}(l+1)^{2}\right),
\end{aligned}
$$

where $\omega_{\chi}=\omega^{2}\left(4 D_{E}\right)^{-1}$. In the expression above, the first term corresponds to the energies of the harmonic oscillator and rigid rotor, the second one reflects corrections to the energy given by anharmonicity and centrifugal distortion, and the last term corresponds to rotation-vibration interaction.

As an exercise, we propose that readers work on some of the following ideas that could improve the presented models:

-Include higher order correction terms in the anharmonic oscillator. (These corrections make possible several other transitions given by the selection rules).

-Include higher order corrections in the rotational Hamiltonian, which would have the same effect as the corrections of the anharmonic oscillator.

-Include molecular interactions, which would take place in a diatomic gas.

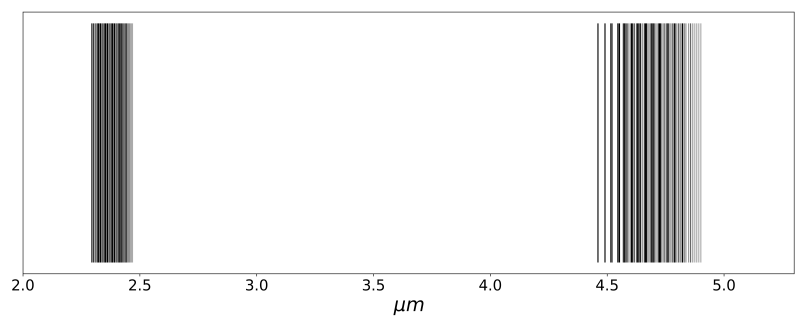

Fig. 1. Computed IR spectrum of the CO molecule. Fundamental and first overtone transitions are present around $2.35 \mu \mathrm{m}$ and $4.67 \mu \mathrm{m}$, respectively. Bands are clearly appreciated.

\section{DISCUSSION}

In the previous section we presented a model considering rotational and vibrational Hamiltonians with a Morse potential as a first approximation for diatomic molecules without molecular interactions. Using equation (14) with the transition values $\Delta n$ and $\Delta l$ of $0, \pm 1, \pm 2$, we obtained the wavelengths corresponding to IR transitions. Our calculations, in spite of not having the amplitude of probability for every transition, expose the complexity of the IR spectrum. We have used equation (14) rather than equation (7) since this model does not include the mixed energy terms.

We apply the results found to the $\mathrm{CO}$ molecule, as shown in Figure 1. Molecular constants for the CO were taken from Le Floch (1991) and are reported in Table 3. Experimental measurements of these constants using a near-IR spectrophotometer were presented by Mina-Camilde et al. (1996).

The CO spectrum shown in Figure 1 illustrates that IR transitions form bands. Our model illustrates how complex it is to obtain the infrared spectrum of heteronuclear diatomic molecules: every transition will depend on the initial state of vibration and rotation, i.e., both quantum numbers $n$ and $l$. Note that the parameters used in the Hamiltonians depend on the moment of inertia, mass and internuclear distance and on the existence of mixed terms in the energy given by equation (15). 
TABLE 3

MOLECULAR CONSTANTS FOR CO

\begin{tabular}{lll}
\hline$B_{e} \times 10^{1}\left(\mathrm{~cm}^{-1}\right)$ & $D_{e} \times 10^{-5}\left(\mathrm{~cm}^{-1}\right)$ & $\alpha_{e} \times 10^{-1}\left(\mathrm{~cm}^{-1}\right)$ \\
0.193128081434 & 0.6121593342 & 0.1750399404 \\
$\beta_{e} \times 10^{-9}\left(\mathrm{~cm}^{-1}\right)$ & $\omega\left(\mathrm{cm}^{-1}\right)$ & $\omega \chi\left(\mathrm{cm}^{-1}\right)$ \\
-0.9316227 & 2169.81259421 & 13.287834373 \\
\hline
\end{tabular}
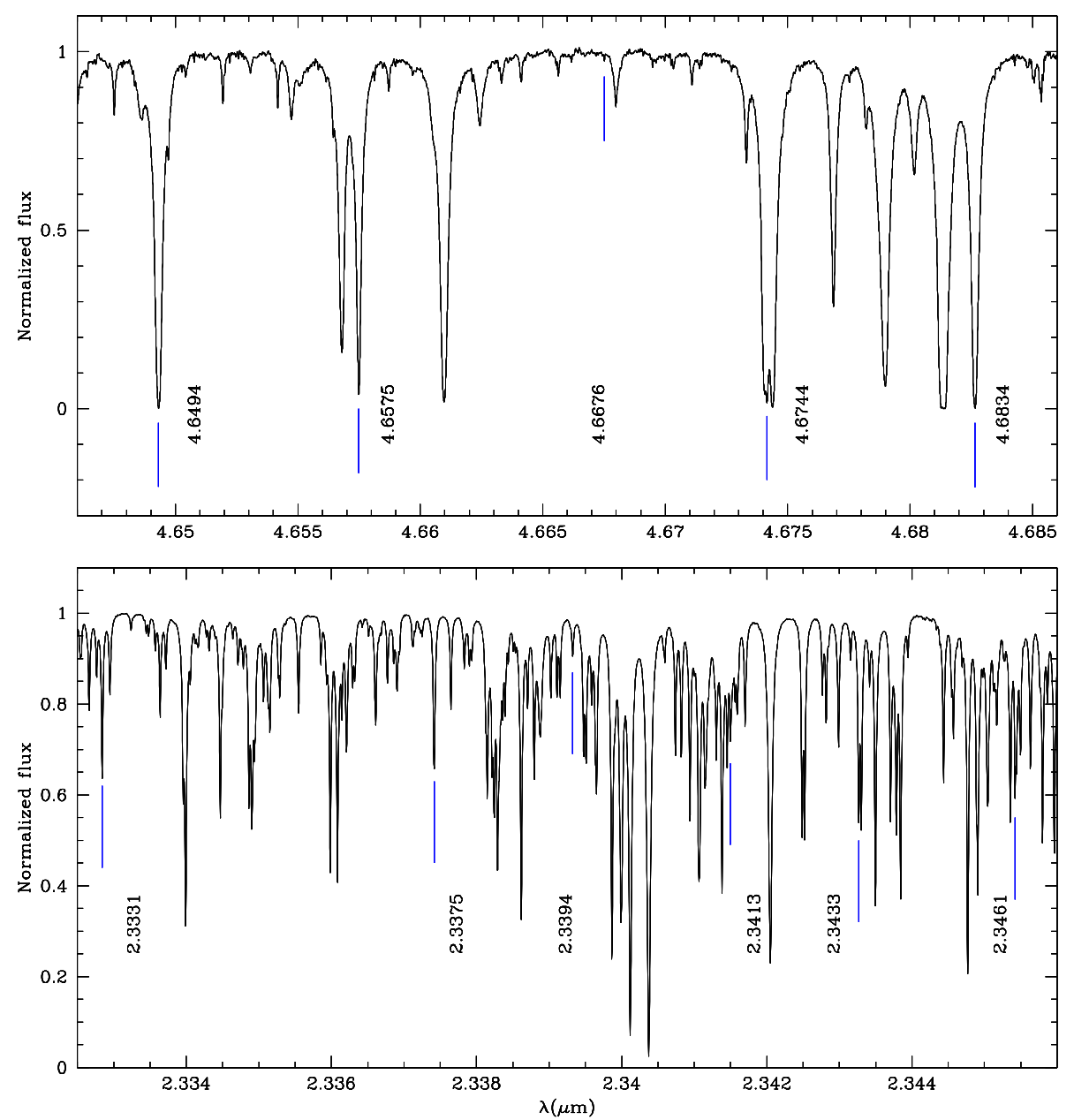

Fig. 2. Line identification. $K_{S}-$ and $M$-band telluric spectra (black) are shown in the bottom and upper panels, respectively. In both panels we show the $\mathrm{CO}$ absorption lines identified with our computed wavelengths (blue). Fundamental mode transitions occur in the $K_{S}$-band. First overtone transitions are present in the $M$-band. The color figure can be viewed online.

We compare our theoretical CO IR wavelengths with those of the high resolution $(R=40000)$ telluric spectrum ${ }^{6}$ created from data made available by

\footnotetext{
${ }^{6}$ These NSO/Kitt Peak FTS IR telluric spectra produced by NSF/NOAO are available at https: //www.eso.org/sci/facilities/paranal/decommissioned/isaac/ tools/spectroscopic_standards.html.
}

the NSO/Kitt Peak Observatory, in the $K_{S}-$ and $M$-bands.

Although the atmosphere spectrum presents multiple absorption lines of $\mathrm{CO}, \mathrm{CO}_{2}$ and $\mathrm{H}_{2} \mathrm{O}$, we have identified several CO lines in the observed telluric spectrum using our computed CO lines as the reference spectrum. This identification was produced 
by means of the $\mathrm{IRAF}^{7}$ task identify of the package onedspec (devoted to reduction and calibration of spectra) reaching an average precision of 0.003 per cent with respect to our theoretical wavelengths. We also made a linear regression between our wavelengths and those identified in the telluric spectrum. The slope of the regression is equal to one, the intercept is zero (with a precision of $10^{-7}$ ) and the $R^{2}$ coefficient is one (with a precision of $10^{-11}$ ). This linear regression shows that our computed CO wavelengths agree with those identified in the telluric spectrum.

Figure 2 illustrates the $K_{S}-$ and $M$ - bands IR telluric spectra, where CO absorption lines are clearly identified with our computed lines. In these regions the absorption features present in the atmosphere are caused by water vapour, carbon dioxide and greenhouse gases.

\section{CONCLUSIONS}

In order to analyse the IR spectra of astronomical sources it is important to identify the telluric features that are produced by the terrestrial atmosphere. In this work we present a quantum mechanics model, useful to obtain analytical expressions for the transition energies of heteronuclear atmospheric molecules. The Hamiltonian used includes the rotation and vibration of diatomic molecules, as well as the interactions between them. We showed that it is possible, with this model, to identify transitions of CO molecule that are present in the $K_{S}-$ and $M-$ bands.
We thank the anonymous referee for valuable corrections and comments, which have enriched this article. We also thank Javier Acevedo for help with the Python graphs and Dr. Neelima Kelkar, for comments. Both are members of the Physics Department of the Universidad de los Andes. AGV and BS also acknowledge financial support to this work by Fondo de Investigaciones de la Facultad de Ciencias de la Universidad de los Andes, Colombia, through Programa de Investigación INV-2019-84-1815 and INV-2019-84-1857. Finally, we thank NSF/NOAO for the NSO/Kitt Peak FTS IR telluric data used here.

\section{REFERENCES}

Atkins, P. \& de Paula, J. 2014, Physical chemistry, (OUP)

Cohen-Tannoudji, C., Diu, B., \& Laloë, F. 1977, Quantum mechanics, Vols 1 \& 2, (New York, NY: John Wiley \& Sons)

Cox, A. 2000, Allen's Astrophysical Quantities, (New York, NY: Springer-Verlag)

Herzberg, G. 1950, Spectra of Diatomic Molecules, (New York, NY: D. van Nostrand Co.)

Kronig, R. 1930, Band Spectra and Molecular Structure, (Cambridge, MA: CUP)

Le Floch, A. 1991, A\&AS, 90, 513

Mina-Camilde, N., Manzanares, C., \& Caballero, J. 1996, JChEd, 73, 804

Rudolf, N., Günther, H., Schneider, P., \& Schmitt, J. 2016, A\&A, 585, 113

Stuart, B. 2004, Infrared Spectroscopy: Fundamentals and Applications, (New York, NY: John Wiley \& Sons Ltd.)

Ulmer-Moll, S., Figueira, P., Neal, J. J., Santos, N. C. \& Bonnefoy, M. 2019, A\&A, 621, 79

Alejandro García-Varela and Beatriz Sabogal: Universidad de los Andes, Departamento de Física, Cra. 1 No. 18A-10, Bloque Ip, A.A. 4976 Bogotá, Colombia (josegarc, bsabogal@uniandes.edu.co).

Daniel Lozano-Gómez: Department of Physics and Astronomy, University of Waterloo, Ontario, N2L 3G1, Canada (dlozanog@uwaterloo.ca).

\footnotetext{
${ }^{7}$ IRAF is a software that was distributed by the National Optical Astronomy Observatories.
} 